\title{
Developmental Changes in Brain Network Hub Connectivity in Late Adolescence
}

\author{
Simon T.E. Baker, ${ }^{1,2,3}$ Dan I. Lubman, ${ }^{1}$ Murat Yücel, ${ }^{2,3}$ Nicholas B. Allen, ${ }^{4,5}$ Sarah Whittle, ${ }^{3}{ }^{\circledR B}$ Ben D. Fulcher, ${ }^{2}$ \\ Andrew Zalesky, ${ }^{3,6}$ and Alex Fornito ${ }^{2,3}$ \\ ${ }^{1}$ Turning Point, Eastern Health and Eastern Health Clinical School, Monash University, Victoria 3065, Australia, ${ }^{2}$ Monash Clinical and Imaging \\ Neuroscience, School of Psychological Sciences and Monash Biomedical Imaging, Monash University, Victoria 3168, Australia, ${ }^{3}$ Melbourne \\ Neuropsychiatry Centre, Department of Psychiatry, The University of Melbourne and Melbourne Health, Victoria 3053, Australia, ${ }^{4}$ Melbourne School of \\ Psychological Sciences, ${ }^{5}$ Orygen Youth Health Research Centre, Centre for Youth Mental Health, and ${ }^{6}$ Melbourne School of Engineering, The University of \\ Melbourne, Victoria 3010, Australia
}

The human brain undergoes substantial development throughout adolescence and into early adulthood. This maturational process is thought to include the refinement of connectivity between putative connectivity hub regions of the brain, which collectively form a dense core that enhances the functional integration of anatomically distributed, and functionally specialized, neural systems. Here, we used longitudinal diffusion magnetic resonance imaging to characterize changes in connectivity between 80 cortical and subcortical anatomical regions over a 2 year period in 31 adolescents between the ages of 15 and 19 years. Connectome-wide analysis indicated that only a small subset of connections showed evidence of statistically significant developmental change over the study period, with $8 \%$ and $6 \%$ of connections demonstrating decreased and increased structural connectivity, respectively. Nonetheless, these connections linked 93\% and $90 \%$ of the 80 regions, respectively, pointing to a selective, yet anatomically distributed pattern of developmental changes that involves most of the brain. Hub regions showed a distinct tendency to be highly connected to each other, indicating robust "rich-club" organization. Moreover, connectivity between hubs was disproportionately influenced by development, such that connectivity between subcortical hubs decreased over time, whereas frontal-subcortical and frontal-parietal hub-hub connectivity increased over time. These findings suggest that late adolescence is characterized by selective, yet significant remodeling of hub-hub connectivity, with the topological organization of hubs shifting emphasis from subcortical hubs in favor of an increasingly prominent role for frontal hub regions.

Key words: adolescence; connectome; development; graph theory; MRI; structural connectivity

\section{Introduction}

The human brain undergoes protracted development that extends into the third decade of life (Giedd et al., 2015). This process involves both progressive and regressive cellular events that refine and integrate anatomically distributed and functionally specialized systems (Tau and Peterson, 2010). Particularly noteworthy are maturational changes during adolescence, a period

\footnotetext{
Received Dec. 12, 2014; revised April 11, 2015; accepted May 6, 2015

Author contributions: S.T.E.B., D.I.L., M.Y., N.B.A., and A.F. designed research; S.T.E.B., N.B.A., and S.W. performed research;S.T.E.B., B.D.F., A.Z., and A.F. contributed unpublished reagents/analytic tools; S.T.E.B., B.D.F., A.Z., and A.F. analyzed data; S.T.E.B. and A.F. wrote the paper.

This research was supported by grants from the Colonial Foundation, the National Health and Medical Research Council [NHMRC (Australia); Program Grant 350241], and the Australian Research Council (ARC; Discovery Grants DP0878136 and DP1092637). S.T.E.B. is supported by an Australian Postgraduate Award. M.Y. is supported by a NHMRC Senior Research Fellowship (1021973). S.W. and A.Z. are supported by NHMRC Career Development Fellowships (1007716 to S.W. and 1047648 to A.Z.). B.D.F. is supported by a NHMRC Early Career Fellowship (1089718). A.F. is supported by an ARC Future Fellowship (FT130100589) and NHMRC Project Grants (1066779 and 1050504).

The authors declare no competing financial interests.

Correspondence should be addressed to Alex Fornito, Monash Clinical and Imaging Neuroscience, 770 Blackburn Road, Clayton, VIC 3168, Australia. E-mail: alex.fornito@monash.edu.

N. B. Allen's present address: Department of Psychology, University of Oregon, Eugene, OR 97403.

DOI:10.1523/JNEUROSCI.5043-14.2015

Copyright $\odot 2015$ the authors $\quad 0270-6474 / 15 / 359078-10 \$ 15.00 / 0$
}

that coincides with heightened vulnerability to mental illness and behavioral problems (Paus et al., 2008). Understanding adolescent brain maturation is therefore critical for identifying neurodevelopmental pathways that confer risk or resilience to psychopathology (Di Martino et al., 2014).

Magnetic resonance imaging (MRI) studies have found that adolescent changes in the organization of large-scale structural and functional neural networks involve the strengthening of long-range connections between distal brain regions (Fair et al., 2007, 2009; Kelly et al., 2009; Dosenbach et al., 2010; Hagmann et al., 2010; Dennis et al., 2013; for review, see Di Martino et al., 2014). Recent studies have suggested that these connections are supported by a collection of spatially distributed and topologically central regions-putative network hubs (Harriger et al., 2012; van den Heuvel et al., 2012; de Reus and van den Heuvel, 2013; van den Heuvel and Sporns, 2013) — and may enhance functional integration (Uddin et al., 2011; Grayson et al., 2014). The maturation of connectivity between hub regions may thus enable the integrated brain function that marks the transition from adolescent to adult patterns of neural activity (Fair et al., 2009).

Despite this view, recent evidence also suggests that hub-hub connectivity may be established early in development, with most 
subsequent developmental change occurring in connections between hubs and topologically peripheral nonhub regions (Hwang et al., 2013; Towlson et al., 2013). For example, one recent study of preterm born infants found that strong connectivity between a core set of hub regions, referred to as a "rich club" (Colizza et al., 2006; van den Heuvel and Sporns, 2011), was present by 30 weeks of gestation, and remained relatively stable until term-equivalent age (Ball et al., 2014). In contrast, connections between hubs and nonhubs underwent the most pronounced development (Ball et al., 2014). This finding is consistent with evidence in the nematode worm Caenorhabditis elegans indicating that rich-club neurons are the earliest to develop (Towlson et al., 2013). In contrast to the adolescent MRI literature, this work suggests that connectivity between hubs may be established early, forming a stable backbone around which additional network elements continue to develop throughout childhood and adolescence. However, a direct, longitudinal analysis of developmental changes in hub connectivity during adolescence is lacking.

Here, we address this gap by comprehensively mapping longitudinal changes in structural connectivity over a 2 year period during mid to late adolescence to determine whether there is any evidence for the selective development of specific types of neural connections. If connectivity between hubs is established early, as has been implied by studies of preterm-born infants (Ball et al., 2014) and model organisms (Towlson et al., 2013), then we expect that adolescent development of these connections should be marginal, and that most change will occur in connections between hubs and nonhubs. If, on the other hand, there is ongoing development of long-range connections that support integrated brain function, as suggested by the adolescent MRI literature (Di Martino et al., 2014), then we expect the most pronounced developmental changes to occur precisely in connections between spatially distributed hub regions.

\section{Materials and Methods}

\section{Participants}

Our analysis included 31 healthy right-handed adolescents (18 male, 13 female) assessed with MRI in the age range of $15.58-17.94$ years (mean, $16.58 ; \mathrm{SD}, 0.54)$ and again a mean of 2.27 years $(\mathrm{SD}, 0.25)$ later in the age range of 17.89-19.96 years (mean, 18.85; SD, 0.45). We focused on this late adolescent developmental epoch because it represents a time that precedes the transition from adolescence to adulthood. Participants were recruited as part of a longitudinal research project investigating biological, psychological, and social risk factors for psychopathology (for further details, see Whittle et al., 2008; Dwyer et al., 2014). From among this community sample, 53 eligible participants were identified for potential inclusion in the current study, with initial eligibility determined on the basis of (1) having completed diffusion- and T1-weighted MRI sequences at both 15-18 and 18-20 years of age and (2) the absence of a lifetime history of a psychiatric diagnosis, as our goal was to investigate normative development of structural connectivity within individuals. We also excluded participants with evidence of a major medical or neurological condition, a contraindication related to medication, a neurological or incidental radiological abnormality detected as part of routine MRI screening by a hospital radiologist, or a history of head injury resulting in loss of consciousness. Based on these criteria, a total of 10 participants (of 53 participants; 19\%) were excluded, leaving 43 eligible participants. As a result of technical issues (e.g., hardware-induced, physiologic, and metallic artifacts) and quality control, diffusion- and T1weighted MRI data of the high standard required for structural connectivity analysis were available for 32 participants (of 43 participants; $74 \%$ ). One left-handed participant was excluded to eliminate the possible effects of handedness, leaving a final sample of 31 adolescents. The final sample had an average Full Scale Intelligence Quotient of 106.71 (SD, 15.88; range, 75-140) at the baseline time point, as measured by an age-appropriate scale of intelligence (Wechsler, 1999, 2003).

\section{MRI acquisition}

MRI was performed on a 3 Tesla system (MAGNETOM Trio, A Tim System, Siemens Medical Solutions) at the Royal Children's Hospital, Melbourne, including diffusion-weighted and T1-weighted sequences. For the high angular resolution diffusion imaging (HARDI) acquisition, 60 gradient-weighted volumes were acquired using a multidirection, twice-refocused spin-echo echoplanar imaging sequence with the following parameters: 60 directions; diffusion-sensitizing gradient (b-value) $=$ $3000 \mathrm{~s} / \mathrm{mm}^{2}$; slice thickness $=2.3 \mathrm{~mm}$; repetition time $(\mathrm{TR})=7300 \mathrm{~ms}$; echo time $(\mathrm{TE})=104 \mathrm{~ms}$; field of view $(\mathrm{FOV})=240 \mathrm{~mm}^{2}$; image matrix $=104 \times 104$; and voxel size $=2.3 \mathrm{~mm}^{3}$ (isotropic). Twicerefocused spin-echo has been shown to reduce eddy current-induced distortion (Reese et al., 2003). In addition, seven T2-weighted (i.e., $\mathrm{b}$-value $=0$; no gradient weighting) volumes were acquired, interspersed throughout the gradient-weighted volumes. For the high-resolution T1weighted acquisition, a three-dimensional magnetization-prepared rapid acquisition gradient echo sequence was used to obtain $176 \mathrm{~T} 1-$ weighted contiguous 0.9 - $\mathrm{mm}$-thick slices. Imaging parameters were as follows: $\mathrm{TR}=1900 \mathrm{~ms}$; TE $=2.24 \mathrm{~ms}$; flip angle $=9^{\circ} ; \mathrm{FOV}=230 \mathrm{~mm}^{2}$; image matrix $=256 \times 256$; voxel size $=0.9 \mathrm{~mm}^{3}$ (isotropic).

\section{MRI processing and connectome mapping}

The following processing pipeline was repeated on baseline and follow-up MRI data for each subject. Raw HARDI and T1-weighted volumes were screened visually for gross artifacts and quantitatively for excessive head movement before being processed using well validated, widely accepted neuroimaging analysis tools, including FreeSurfer (Fischl, 2012), FMRIB Software Library (Jenkinson et al., 2012), MRtrix (Tournier et al., 2012), and Brain Connectivity Toolbox (Rubinov and Sporns, 2010; https://sites.google.com/site/bctnet/). Networks were visualized using BrainNet Viewer (Xia et al., 2013; www.nitrc.org/projects/bnv/), and NeuroMArVL (Monash Adaptive Visualization Lab; http://marvl.infotech.monash.edu.au/).

The T1-weighted volume was parcellated into 33 cortical (Desikan et al., 2006) and 7 subcortical (Fischl et al., 2002) homologous regions in each hemisphere, totaling 80 distinct brain regions. The inverse transformation matrix from the linear alignment of the mean b0 volume to the T1-weighted volume in Talairach space was applied to the parcellation to align the cortical and subcortical regions to diffusion space (Jenkinson et al., 2002). Non-negativity constrained super-resolved spherical deconvolution-better known as constrained spherical deconvolution (CSD) - was used to estimate the distribution of fiber orientations at each voxel of the HARDI volume (Tournier et al., 2012). The advantages of CSD include improved characterization of complex fiber distributions within each voxel, thereby reducing spurious fiber dispersion, false positives, and false negatives when fiber tracking (Tournier et al., 2008; Jeurissen et al., 2011; Ramirez-Manzanares et al., 2011). Whole-brain tractography using a probabilistic fiber-tracking algorithm was performed on CSD data to obtain a computer-generated "tractogram" comprised of 1 million streamlines that collectively estimate the trajectories of axonal pathways in the brain (Tournier et al., 2012). Probabilistic tractography combined with CSD has been shown to be useful for resolving crossing fibers and for reconstructing axonal pathways that correspond well with known white matter anatomy (Jeurissen et al., 2011; Tournier et al., 2012).

The parcellation and tractogram were combined to produce an $80 \times$ 80 weighted, undirected connectivity matrix. In this matrix, each $[i, j]$ matrix element represented the number of streamlines intersecting regions $i$ and $j$, which we derived by filtering the tractogram to include only streamlines whose points of propagation intersected two or more different regions. A streamline intersecting more than two regions was assumed to connect the pair of regions that were maximally separated according to the Euclidean distance calculated using the center of gravity of each region. To avoid the inclusion of spurious streamlines crossing the medial longitudinal fissure, interhemispheric streamlines were restricted to those with at least one point of propagation located in a mask created by the mean dilation of the corpus callosum.

To further reduce the number of potentially spurious connections, region pairs with a streamline count less than $T=38$ were set to 0 . The 
value of $T$ was chosen as the maximum threshold that could be applied to the connectivity matrices while ensuring that each matrix formed a single, connected component (i.e., a fully connected network; as described by Lynall et al., 2010), which is a reasonable assumption for the brain. Secondary analyses indicated that results were robust under variations of $T$.

Within each individual dataset, matrix elements, each encoding the number of streamlines connecting a unique pair of brain regions, were converted to a streamline density value, calculated as the number of streamlines over the total volume (in cubic millimeters) of the two connected brain regions. Using this streamline density measure mitigates the potential for larger brain regions to have higher connectivity simply because they possess a greater surface area (Hagmann et al., 2010; van den Heuvel and Sporns, 2011). The primary analyses were performed using these participant-specific streamline density matrices. Secondary analyses were performed using participant-specific mean fractional anisotropy (FA) matrices, wherein each matrix element encoded the mean of FA for voxels traversed by at least one of the set of streamlines connecting a unique pair of brain regions.

\section{Analyses}

Our analyses used tools from graph theory (Bullmore and Sporns, 2009; Fornito et al., 2013) to understand brain network organization. In graph theory, brain networks are represented as collections of nodes (brain regions) connected by edges (structural connections). In our primary analyses, edge weights represented the streamline density of connections between nodes. The following describes our pipeline for characterizing developmental changes in relation to hub connectivity.

Network-based statistic. Our first goal in the analysis pipeline was to identify structural connections showing significant effects of development across the entire connectome in a regionally unbiased way. To this end, we used the Network Based Statistic (NBS; Zalesky et al., 2010a) to identify subsets of edges showing either greater connectivity (i.e., increased streamline density) at baseline compared with follow-up or vice versa. The NBS is a validated nonparametric statistical approach for controlling familywise error (FWE) in connectome-wide analyses. Compared with conservative FWE controlling procedures (e.g., Bonferroni and false discovery rate), the NBS offers greater statistical power to identify statistically significant effects in network analyses (Zalesky et al., 2010a, 2011).

For each connection, we computed a one-tailed paired-samples $t$ test comparing the magnitude of the edge weight at each individual element of the connectivity matrix between baseline and follow-up. We then applied a threshold of $p=0.05$ to the resulting $t$ statistic matrix to yield a binarized matrix of suprathreshold connections. A breadth first search (Ahuja et al., 1993) was then used to identify any connected components (i.e., subnetworks) in this matrix, and the number of connections in each component (i.e., component size) was computed. In this context, a connected component refers to a collection of nodes that is linked by a set of suprathreshold edges.

To generate an empirical null distribution for evaluating the statistical significance of the observed component sizes, the two levels (baseline and follow-up) of the fixed factor (time) were shuffled 5000 times while constraining the random factor (subject) so that each individual contributed only one observation to each level of the permuted within-subject factor. This constraint accounts for the fact that repeated measurements on the same individual will be correlated and cannot be considered exchangeable under the null hypothesis (Suckling and Bullmore, 2004). The maximal component size was retained at each iteration, and these values were used to generate an empirical null distribution of maximal component sizes, against which the observed component sizes were evaluated. This procedure ensures FWE correction of the resulting component-wide $p$ values. Components with component-wide $p<0.05$ were considered statistically significant.

Rich-club analysis. The rich-club phenomenon refers to the tendency of high-degree nodes (e.g., hub regions) to be highly connected to each other, more so than expected by chance (Colizza et al., 2006).
Rich-club organization was examined using a mean streamline density matrix, calculated as the average value at each edge of streamline density matrices at follow-up across all subjects. The follow-up data were used for this analysis because they represent the network in its most mature configuration. We note that rich-club behavior was also observed in the baseline group-averaged streamline density matrix.

The tendency of hubs to be highly connected to each other can be measured with the rich-club coefficient. We calculated the weighted richclub coefficient, $\phi^{w}(k)$, in the group-averaged network according to the following equation (Opsahl et al., 2008; van den Heuvel and Sporns, 2011):

$$
\phi^{w}(k)=\frac{W_{>k}}{\sum_{l=1}^{E_{>k}} w_{l}^{\text {ranked }}},
$$

where $W_{>k}$ represents the sum of weights on edges within the subgraph defined by retaining only nodes with degree $>k, E_{>k}$ is the number of edges present in the subgraph defined by retaining only nodes with degree $>k$, and $w^{\text {ranked }}$ is a vector of ranked (highest to lowest) edge weights taken from the entire network. This equation thus expresses rich-club organization as a ratio between the total weight of links between nodes with degree $>k$, and the total weight of the same number of links taken from the strongest (most highly weighted) connections in the network.

Having computed the rich-club coefficient for each $k$ in our observed data, we then calculated the rich-club coefficient, $\phi_{\text {rand }}^{w}(k)$, for each of 1000 randomized networks. Randomized networks were constructed by shuffling the weighted links in the group-averaged network while preserving the degree distribution (Maslov and Sneppen, 2002; Rubinov and Sporns, 2010). At each level of $k$, the normalized rich-club coefficient, $\phi_{\text {norm }}^{w}(k)$, was computed as the ratio between the observed rich-club coefficient, $\phi^{w}(k)$, and the mean of $\phi_{\text {rand }}^{w}(k)$ across the 1000 randomized networks, $\left\langle\phi_{\text {rand }}^{w}(k)\right\rangle$, as follows:

$$
\Phi_{\text {norm }}^{w}(k)=\frac{\phi^{w}(k)}{\left\langle\phi_{\text {rand }}^{w}(k)\right\rangle} .
$$

The ensemble of 1000 randomized networks also yielded an empirical null distribution of $\phi_{\mathrm{rand}}^{w}$ across the range of $k$, which was used to determine whether $\phi^{w}(k)$ was significantly greater than $\phi_{\text {rand }}^{w}(k)$ at each level of $k$ (one-tailed, $p<0.05$ ). Comparison with respect to this null model is crucial to ensure that any apparent rich-club organization in the network significantly exceeds levels expected by chance. We focus here on the weighted rich-club coefficients computed relative to randomized networks characterized by the same degree sequence as the observed network, as in van den Heuvel and Sporns (2011), but note that rich-club behavior was also observed relative to randomized networks with preserved topology but randomized link weights, and in the unweighted network relative to randomized networks with preserved degree sequence.

\section{Results}

\section{Changes in structural connectivity over time}

We first set out to identify subsets of structural connections showing statistically significant increases and decreases in streamline density from baseline to follow-up. The group average connection density (i.e., the proportion of all possible connections actually present) for each time point was 33\% (SD, 2\%), suggesting that any developmental changes occurring between these time points predominantly involved the redistribution of connectivity weights, rather than large-scale addition or removal of connections.

In the analysis of connections showing significant decreases in streamline density over time, the NBS identified a subnetwork comprising 248 edges ( $8 \%$ of possible connections) linking 74 nodes ( $93 \%$ of the 80 regions; FWE corrected, $p<0.001$ ). In contrast, the NBS subnetwork of connections showing significant increases in streamline density over time comprised 185 edges 

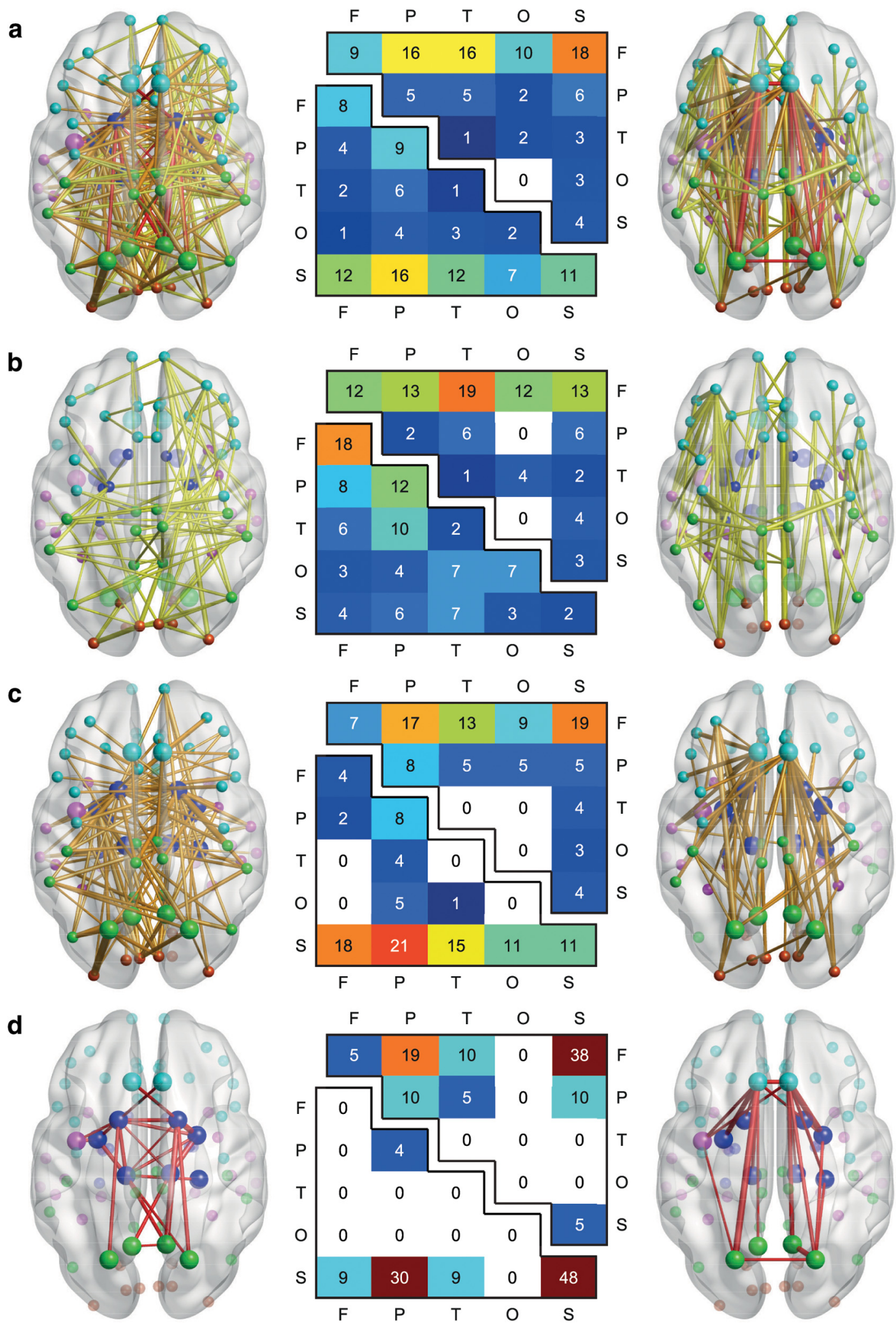

Figure 1. Developmental changes in structural connectivity. $\boldsymbol{a}$ illustrates the anatomical arrangement of the subset of edges identified by the NBS (Zalesky et al., 2010a) as showing significant decreases (left) and increases (right) in streamline density over time, with the thickness of each connection weighted by its associated one-tailed $t$ test statistic (FWE corrected, $p<0.05$ ). Edge color represents connection type: peripheral (yellow), feeder (orange), and hub-hub (red), with larger nodes corresponding to hub regions. Node color represents the assignment of each region of interest to one of five broad anatomical divisions: frontal (cyan), parietal (lime), temporal (magenta), occipital (orange-red), or subcortical (blue). The center panel illustrates the anatomical distribution of the developmental decreases (lower triangular matrix) and increases (upper triangular matrix) in connectivity based on the classification of edges according to the anatomical divisions they interconnected. The values in these matrices represent relative proportions, calculated as the ratio between (1) the frequency of edges linking each pair of divisions and (2) the total number of edges belonging to the two categories. Illustrated in $\boldsymbol{b}-\boldsymbol{d}$ are peripheral, feeder, and hub- hub connections, respectively, extracted from the NBS subnetworks in $\boldsymbol{a}$. 
( $6 \%$ of possible connections) linking 72 nodes ( $90 \%$ of the 80 regions; FWE corrected, $\left.p=6 \times 10^{-4}\right)$. In both of these cases, the proportion of connections showing significant developmental effects was relatively small, but these connections spanned a large proportion of the 80 regions. Figure $1 a$ illustrates the anatomical arrangement of the subset of connections identified by the NBS as showing significant decreases and increases in streamline density over time, with the thickness of each connection weighted by its associated one-tailed $t$ test statistic (FWE corrected, $p<0.05$ ).

These NBS results were robust to variations of the streamline count threshold, T. Specifically, a significant subnetwork showing decreased streamline density over time (FWE corrected, $p<$ 0.05 ) was identified for each threshold in the range $5 \leq T \leq 38$. These networks varied in size between 248 and 354 (8\% and 11\%) edges, and 73 and 76 (91\% and 95\%) nodes. Significant subnetworks showing increased streamline density over time (FWE corrected, $p<0.05$ ) were also identified for thresholds in the range $5 \leq T \leq 38$, with the number of edges varying between 184 and $234(6 \%$ and $7 \%)$, and the number of nodes between 72 and 77 (90\% and $96 \%)$.

The NBS results were also robust to variations of the primary threshold. Changing this threshold to $p=0.025$ yielded similar, albeit smaller, subnetworks: 166 connections (5\%; linking 67 nodes) showed significantly decreased streamline density over time (FWE corrected, $p<0.001$ ), whereas 118 connections (4\%; linking 64 nodes) showed significantly increased streamline density over time (FWE corrected, $p<0.001$ ).

\section{Anatomical distribution of structural connectivity changes}

To understand the anatomical distribution of the connections showing significant developmental effects, we assigned each region to one of the following five broad divisions: frontal, parietal, temporal, occipital, or subcortical. For each NBS subnetwork, we then examined the relative frequency of edges linking each pair of divisions. As shown in Figure $1 a$, connections in the subnetwork showing significant decreases in streamline density over time typically involved subcortical regions, including edges classified as parietal-subcortical (16\%), frontal-subcortical (12\%), temporal-subcortical (12\%), and subcortical-subcortical (11\%). In contrast, connections in the subnetwork showing significant increases in streamline density over time typically involved frontal regions, including edges classified as frontal-subcortical (18\%), frontal-parietal (16\%), frontal-temporal (16\%), and frontalfrontal (9\%; Fig. 1a).

We also compared the NBS subnetworks on the length of their connections. As a proxy for the physical length of connections, we computed the Euclidean distance between the centroids of connected brain regions. The connection distance of edges in the subnetwork showing increased streamline density over time was greater than in the subnetwork showing decreased streamline density over time $\left(t_{(448)}=3.40, p=7 \times 10^{-4}\right)$, suggesting that developmental increases of connectivity were more likely to occur in long-distance connections.

\section{Rich-club organization}

Figure $2 a$ shows the normalized rich-club coefficients, $\Phi_{\text {norm }}^{w}(k)$, for the follow-up group-averaged streamline density-weighted network. Weighted rich-club coefficients, $\phi^{w}(k)$, were significantly greater than those derived from randomized networks, $\phi_{\text {rand }}^{w}(k)$, for a range of $k$ from 30 to 72 (one-tailed, $p<0.05$ ), indicating robust rich-club organization in the network. Thus, brain network hubs showed a significant tendency to be highly
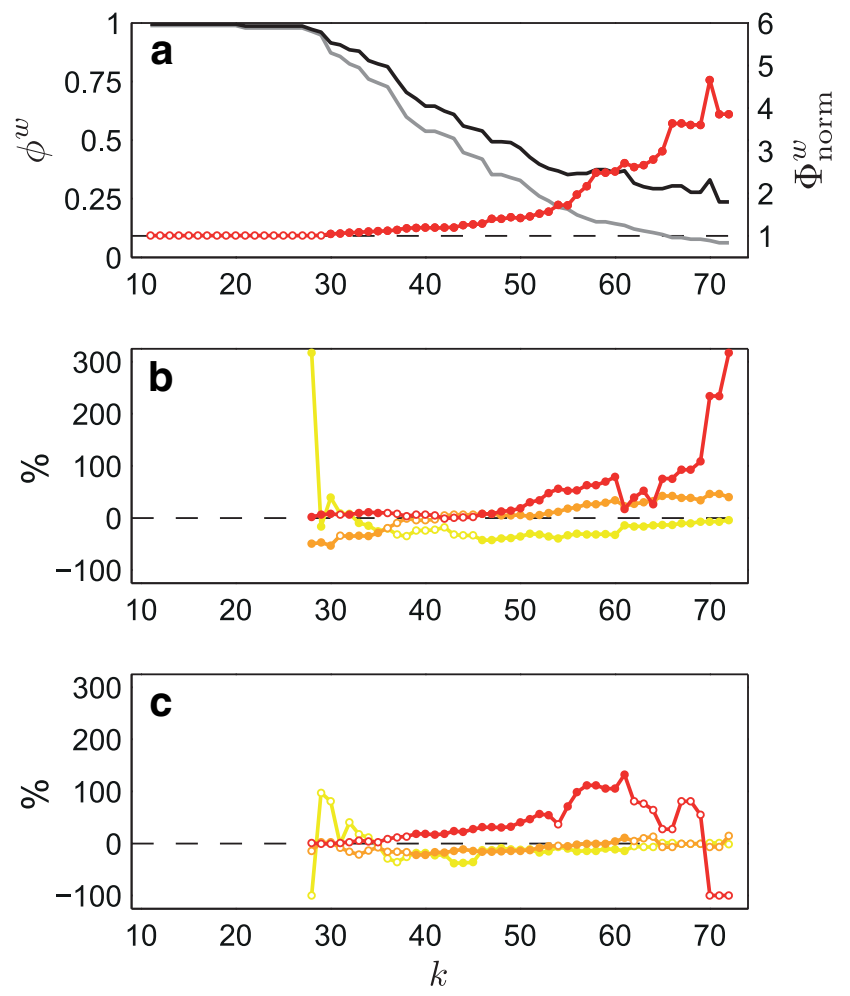

Figure 2. Rich-club curves and magnitude of developmental changes in peripheral, feeder, and hub-hub connections. a shows the normalized rich-club coefficients, $\Phi_{\text {norm }}^{w}(k)$ (red), for the follow-up group-averaged streamline density-weighted network. Weighted rich-club coefficients, $\phi^{w}(k)$ (black), were found to be significantly greater than those derived from randomized networks, $\phi_{\text {rand }}^{w}(k)$ (gray), for a range of $k$ from 30 to 72 (one-tailed, $p<0.05$; filled/solid red markers), indicating robust rich-club organization in the network. $\boldsymbol{b}$ and $c$ show the percentage difference between observed and expected proportions at each level of $k$ for each connection type [peripheral (yellow), feeder (orange), and hub- hub (red)] in the subnetwork showing decreased streamline density over time $(\boldsymbol{b})$ and the subnetwork showing increased streamline density over time $(\boldsymbol{c}) \cdot \chi^{2}$ analysis of the difference between the observed and expected proportions (also at each level of $k$ ) revealed a significant effect of connection type $(p<0.05$; filled/solid markers) in both the subnetwork showing decreased streamline density over time (for each $k$ in the range $27<k<36$ except $k=31$, and $45<k<73 ; \boldsymbol{b}$ ) and the subnetwork showing increased streamline density over time (for each $k$ in the range $30<k<$ 62 except $k=54 ; c)$. As can be seen, a disproportionate number of hub-hub connections showed developmental changes, particularly at levels of $k$ that coincide with the topological rich-club effect $(\boldsymbol{a})$.

connected to each other, forming an anatomical backbone for functional integration of spatially distributed brain regions (van den Heuvel and Sporns, 2013).

\section{Hub classification and connection types}

Nodes with a high degree $(k>1$ SD above the mean, where the mean is 45.55 and SD is 13.91) were classified as network hubs, in accordance with prior work (van den Heuvel and Sporns, 2011). Fourteen such network hubs were identified, and included regions in bilateral superior-frontal and superior-parietal cortex, precuneus, caudate, putamen, and thalamus, as well as left insula and right hippocampus. Defining hub regions based on the follow-up group-averaged streamline density-weighted network did not affect the findings because the set of hubs at each time point was identical, except for one region.

Based on this definition of hub regions, edges identified by the NBS were classified as one of the following connection types: peripheral connections, which link nonhubs; feeder connections, 

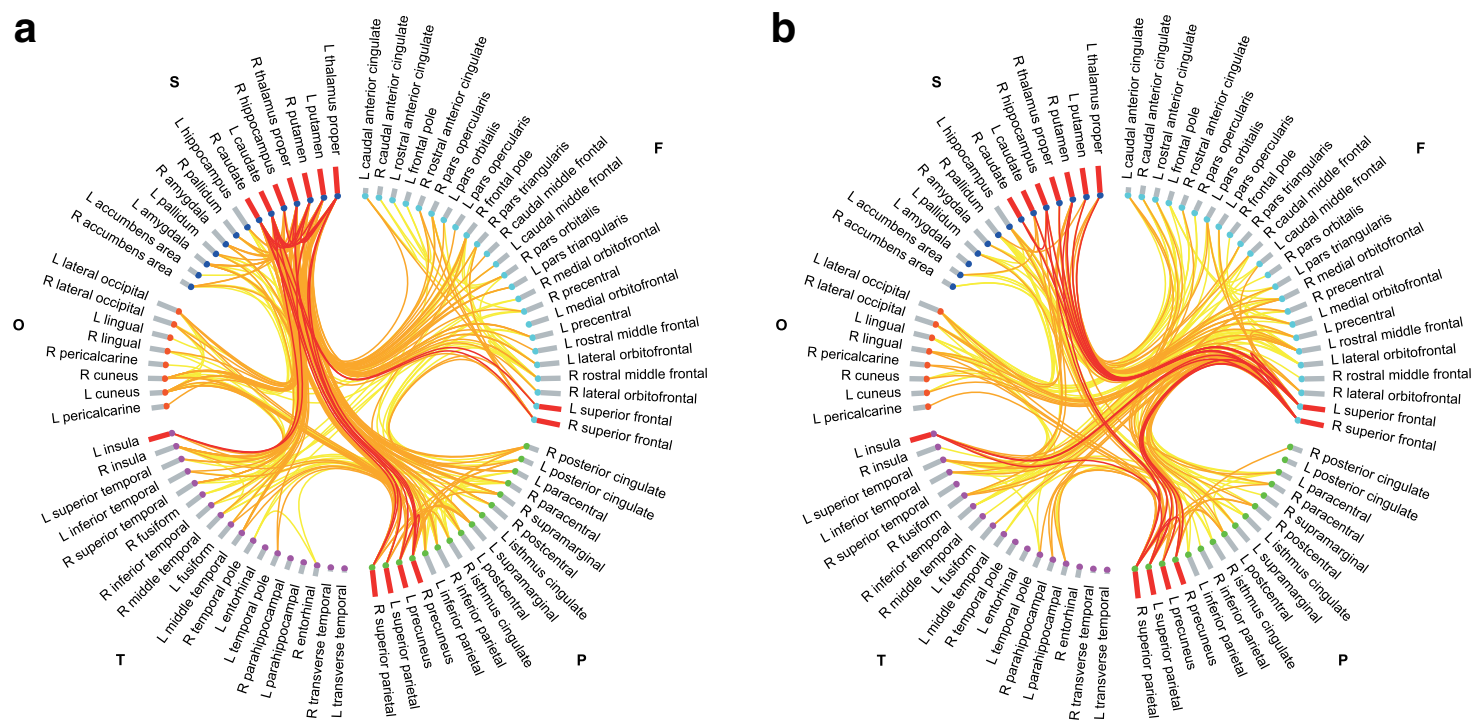

Figure 3. Connectograms of developmental decreases and increases in streamline density are shown in $\boldsymbol{a}$ and $\boldsymbol{b}$, respectively, with links colored by connection type: peripheral (yellow), feeder (orange), and hub- hub (red). Regions of interest are grouped according to broad anatomical divisions [i.e., frontal (cyan), parietal (lime), temporal (magenta), occipital (orange-red), or subcortical (blue)] and are further ordered by degree (bar height is proportional to degree), with bars colored in red corresponding to hub regions. F, Frontal; L, left; 0 , occipital; $P$, parietal; R, right; S, subcortical; T, temporal.

which link nonhubs and hubs; and hub-hub connections, which link two hubs. The proportions of edges showing decreased streamline density over time that were classified as peripheral, feeder, or hub-hub were $36 \%, 55 \%$, and $9 \%$, respectively. Among edges showing increased streamline density over time, $48 \%, 41 \%$, and $11 \%$ were classified as peripheral, feeder, and hub-hub connections, respectively.

These raw proportions suggest that developmental changes in hub-hub connections are relatively minor during late adolescence. However, there generally tends to be fewer hub-hub connections in the brain compared with feeder and peripheral connections, and this difference will bias analyses of raw proportions in the NBS subnetworks. To control for this bias, we performed $\chi^{2}$ analyses to test for differences in the distribution of distinct connection types in the NBS subnetworks. At each level of $k$, we classified nodes with degree $>k$ as hubs, then computed the following: (1) the observed proportion of each connection type (peripheral, feeder, and hub-hub) in the NBS subnetwork; and (2) the expected proportion of each connection type (based on the actual proportion of each connection type in the groupaveraged network). $\chi^{2}$ analysis of the difference between these proportions (also at each level of $k$ ) revealed a significant effect of connection type $(p<0.05)$ in both the subnetwork showing decreased streamline density over time (for each $k$ in the range $27<k<36$, except for $k=31$, and $45<k<73$ ), and the subnetwork showing increased streamline density over time (for each $k$ in the range $30<k<62$, except for $k=54$ ). These results are illustrated in Figure 2, $b$ and $c$, which plots the percentage difference between observed and expected proportions at each level of $k$ for each connection type in the subnetwork showing decreased streamline density over time (Fig. $2 b$ ) and the subnetwork showing increased streamline density over time (Fig. $2 c$ ). As can be seen, a disproportionate number of hub-hub connections showed developmental changes, particularly at levels of $k$ that coincide with the topological rich-club effect (Fig. 2a).

\section{Anatomical distribution of changes in peripheral, feeder, and} hub-hub connectivity

Figures 1 and 3 illustrate the anatomical distribution of developmental changes in peripheral, feeder, and hub-hub connectivity. As shown in Figure 1b, peripheral connections in the subnetwork showing significant decreases in streamline density over time were distributed relatively evenly throughout the network-18\% were frontal-frontal, $12 \%$ were parietal-parietal, and $10 \%$ were parietal-temporal-and peripheral connections between frontal and other anatomical divisions were a feature of the subnetwork showing significant increases in streamline density over time. Figure $1 c$ shows that among feeder connections, the most prominent developmental decreases in streamline density were between subcortical and other anatomical divisions (e.g., parietal-subcortical, 21\%), and the most prominent developmental increases in streamline density were between frontal and other anatomical divisions (e.g., frontal-subcortical, 19\%). As shown in Figure $1 d$, nearly half the hub-hub connections in the subnetwork showing decreased streamline density over time were subcortical-subcortical (48\%), while nearly one-third were parietal-subcortical (30\%). In addition, hub-hub connections in the subnetwork showing increased streamline density over time were predominantly frontal-subcortical (38\%) and frontal-parietal (19\%; Fig. 1d). Connectograms illustrating the developmental decreases and increases in streamline density for peripheral, feeder, and hub-hub connections are shown in Figure 3, $a$ and $b$.

\section{The effect of connectivity weight and parcellation}

Further analyses were performed to examine whether the findings were sensitive to the specific measure of connectivity weight or the choice of parcellation. Regarding the former, we repeated our analyses using mean FA-weighted networks, wherein each matrix element encoded the mean of FA for voxels traversed by at least one of the set of streamlines connecting a unique pair of brain regions. Similar to streamline density-weighted networks, 170 connections (5\%) linking 69 nodes showed significantly decreased mean FA (FWE corrected, $p=0.028$ ), and 254 connec- 
tions ( $8 \%)$ linking 76 nodes showed significantly increased mean FA (FWE corrected, $p=6 \times 10^{-4}$ ). There was rich-club organization in both baseline and follow-up group-averaged mean FAweighted networks. The disproportionate number of hub-hub connections was evident in the subnetwork showing increases in mean FA over time, with $\chi^{2}$ analyses revealing a significant effect of connection type $(p<0.05)$ for each $k$ in the range $35<k<70$, but no such pattern was apparent in the subnetwork showing decreases in mean FA over time, suggesting that the developmental increases of hub connectivity are more robust than decreases to the way in which connectivity weight is defined.

To ensure that the findings were not driven by the chosen parcellation scheme (Fischl et al., 2002; Desikan et al., 2006), we repeated the analyses using a random parcellation comprising 530 nodes (265 per hemisphere) of approximately equal volume, generated according to the methods described by Zalesky et al. (2010b) and Fornito et al. (2010, 2011). For streamline densityweighted networks, 5889 connections (4\%) linking all 530 nodes showed significantly decreased streamline density (FWE corrected, $p<0.001$ ), and 4730 connections (3\%) linking 528 nodes showed significantly increased streamline density (FWE corrected, $p=0.035)$. Similarly, for mean FA-weighted networks, 5732 connections (4\%) linking all 530 nodes showed significantly decreased mean FA (FWE corrected, $p=0.014$ ), and 6151 connections (4\%) linking all 530 nodes showed significantly increased mean FA (FWE corrected, $p=0.001$ ). Figure $4 a$ illustrates the anatomical location of nodes classified as hubs (nonhubs) in the follow-up group-averaged streamline densityweighted network based on the 530-node parcellation according to the high-degree cutoff $k>1$ SD above the mean (mean, 368.68; $\mathrm{SD}, 79.94)$. The localization of hubs to association cortex and subcortical areas is consistent with the spatial distribution of hub nodes obtained using the 80 -node parcellation (Fig. $2 d$ ). Networks based on the 530-node parcellation showed robust richclub organization. Figure $4 b$ shows this effect for the follow-up group-averaged streamline density-weighted network. Similar results were obtained for the baseline group-averaged streamline density-weighted network, and both baseline and follow-up group-averaged mean FA-weighted networks. As shown in Figure $4, c$ and $d$, developmental changes - particularly increases - in streamline density were predominantly related to hub-hub connectivity. Developmental increases in mean FA also showed a preference for changes in hub-hub connectivity.

\section{Discussion}

This study examined longitudinal changes in structural connectivity of brain network hub regions during late adolescence. Our findings indicate that this period is characterized by selective, yet significant remodeling of connections between spatially distributed hub regions. The most prominent changes in hub-hub connectivity over time involved decreases between subcortical regions and increases between frontal and subcortical regions. These findings support the hypothesis that refinement of hubhub connectivity continues into late adolescence. Further, they suggest that this development is characterized by a relative redistribution of the topological importance of distinct classes of hub regions, in which there is a gradual de-emphasis of subcortical connections and an increasing emphasis on connections between frontal and subcortical areas.

Our findings suggest that late adolescence is characterized by a complex combination of selective, yet anatomically distributed progressive and regressive changes, such that only a small proportion of connections showed evidence of statistically signifi-
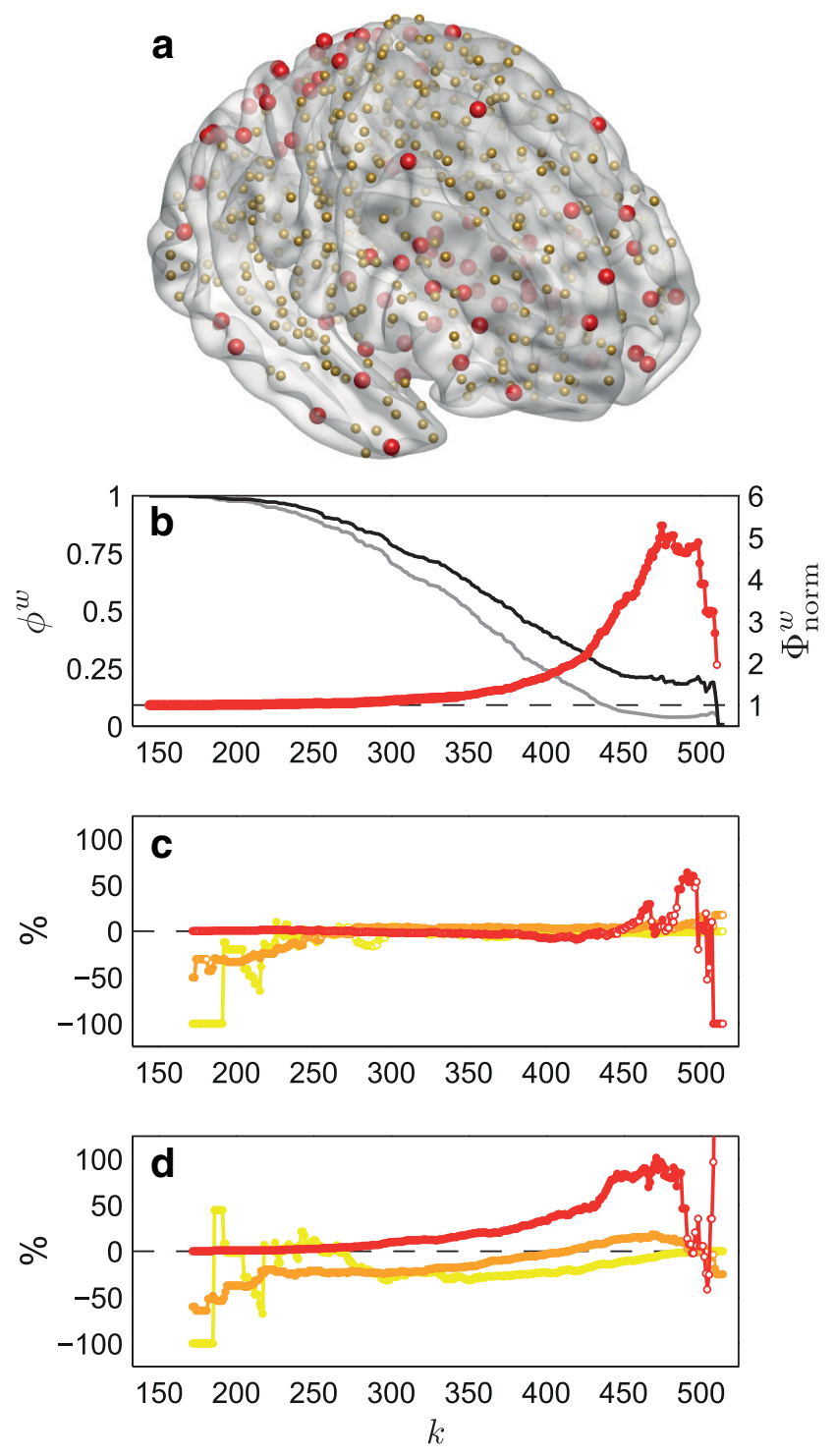

Figure 4. The effect of parcellation scale on developmental changes in structural connectivity. $\boldsymbol{a}$ illustrates the anatomical location of nodes classified as hubs (red) and nonhubs (gold) in the follow-up group-averaged streamline density-weighted network based on the 530-node parcellation. $\boldsymbol{b}$ shows the normalized rich-club coefficients, $\Phi_{\text {norm }}^{w}(k)$ (red), for this network. Weighted rich-club coefficients, $\phi^{w}(k)$ (black), were found to be significantly greater than those derived from randomized networks, $\phi_{\text {rand }}^{w}(k)$ (gray), for each $k$ in the range $143<k<$ 170 and $172<k<510$ (one-tailed, $p<0.05$; filled/solid red markers), indicating robust rich-club organization in the network. $\boldsymbol{c}$ and $\boldsymbol{d}$ show the percentage difference between observed and expected proportions at each level of $k$ for each connection type [peripheral (yellow), feeder (orange), and hub- hub (red)] in the subnetwork showing decreased streamline density over time $(\boldsymbol{c})$ and the subnetwork showing increased streamline density over time $(\boldsymbol{d}) . \chi^{2}$ analysis of the difference between the observed and expected proportions (also at each level of $k$ ) revealed a significant effect of connection type ( $p<0.05$; filled/solid markers) in both the subnetwork showing decreased streamline density over time (for the majority of $k$ in the range $171<k<503 ; c$ ) and the subnetwork showing increased streamline density over time (for each $k$ in the range $171<k<491$ and $508<k<515 ; \boldsymbol{d}$ ). As can be seen, developmental changes - particularly increases - in streamline density were predominantly related to hubhub connectivity, particularly at levels of $k$ that coincide with the topological rich-club effect $(\boldsymbol{b})$.

cant developmental change, but these connections linked regions located throughout the brain. The fact that this type of change is occurring during mid to late adolescence is further evidence that significant neurodevelopmental processes, with potentially important implications for behavior and mental health, are still occurring during this phase of life. Indeed, our observation that 
structural hub-hub connectivity undergoes significant development during adolescence is consistent with previous studies demonstrating that significant remodeling of functional connectivity between hubs occurs between childhood and adulthood (Uddin et al., 2011; Grayson et al., 2014). Thus, unlike the developmental window between 30 and 40 weeks of gestation, which is an important period for the development of connections between (rich-club) hub regions and topologically peripheral regions (i.e., feeder connections; Ball et al., 2014), late adolescence appears to be an important period for the refinement of hub-hub connectivity. It is thus possible that distinct connection types may undergo development at different rates, and during different developmental epochs. More specifically, hub-hub connectivity may emerge early to act as a scaffold upon which other regions are added to the network. As the network becomes more established, it may undergo further refinement, which, in adolescence, is characterized by an augmentation of hub-hub connectivity to support the increasing levels of functional integration thought to characterize this developmental period. This is not to say that connections involving nonhub regions do not develop in childhood and adolescence; indeed, our own data indicate that, in absolute terms, most of the connections showing developmental changes involved nonhubs. However, our findings point to a disproportionate number of hub-hub connections undergoing developmental change when considered relative to the proportion of each connection type in the brain.

Subnetworks of connections showing decreased and increased connectivity (i.e., streamline density) over time had distinct anatomical distributions. Specifically, the connectivity of frontal regions tended to increase with development, whereas subcortical connectivity tended to decrease. This trend was observed at both the connectome-wide level and specifically with respect to hub-hub connections. Increased connectivity between frontal and subcortical hubs was particularly prominent. These findings are consistent with a large body of work indicating that the frontal cortex and its associated white matter undergo a protracted period of development extending into the third decade of life (Yakovlev and Lecours, 1967; Huttenlocher, 1979; Giedd et al., 2015), and with evidence that connectivity between the frontal cortex and striatum (i.e., caudate and putamen) undergoes marked developmental change during adolescence (Sowell et al., 1999; Paus et al., 2001; Liston et al., 2006; Hwang et al., 2010; Schmithorst and Yuan, 2010). Our findings may thus reflect a developmental process known as "frontalization," whereby the frontal lobes become increasingly dominant in planning, organizing, and regulating thought and behavior, enabling the integration of information from diverse parts of the brain to support complex behavior (Lewis and Todd, 2007). Developmental changes in the link between frontal and subcortical regions may have particular relevance for understanding risk taking (Spear, 2000; Steinberg, 2005) and depression (Davey et al., 2008) during adolescence. It is well known that subcortical regions, which develop early in life, are primarily responsible for the initiation of affective/emotional and reward-seeking processes (Panksepp, 1998). Decreasing connectivity between subcortical areas and increasing connectivity with frontal cortex may render these regions less autonomous and more receptive to top-down control during the transition from adolescence to adulthood, thus coinciding with a higher degree of behavioral regulation.

It is well known that adolescence is associated with substantial myelination of long-range axonal pathways (Benes et al., 1994). Our data are consistent with these observations and suggest that myelination of hub-hub connectivity may be particularly pro- nounced in late adolescence. However, caution is warranted when inferring the physiological basis of changes in connectivity measures derived from diffusion MRI, and it is possible that changes in fiber trajectory, organization, density, or other parameters related to axonal integrity may also affect the measures examined in our analysis (Paus, 2010; Fornito et al., 2013; Jones et al., 2013).

Our sample size precluded an analysis of sex differences, which are known to influence brain development (Giedd et al., 2015). Replication and extension of our findings in a larger sample are thus required. Understanding how developmental changes in hub connectivity affect network topology and dynamics will also be an important goal for future research.

In summary, we report evidence for selective, yet anatomically distributed progressive and regressive changes in structural connectivity in late adolescence. The specific pattern of developmental changes is most pronounced for connectivity between hub regions, and is consistent with an increasingly prominent role for frontal hub regions, coupled with a decreasing emphasis on the connectivity of subcortical structures. Understanding how derailment of these developmental processes contributes to risk for psychopathology may assist efforts to predict disease vulnerability and target early interventions, given that many brain disorders are associated with hub pathology (van den Heuvel et al., 2013; Crossley et al., 2014; Fornito et al., 2015).

\section{References}

Ahuja RK, Magnanti TL, Orlin JB (1993) Network flows: theory, algorithms, and applications. Upper Saddle River, NJ: Prentice Hall.

Ball G, Aljabar P, Zebari S, Tusor N, Arichi T, Merchant N, Robinson EC, Ogundipe E, Rueckert D, Edwards AD, Counsell SJ (2014) Rich-club organization of the newborn human brain. Proc Natl Acad Sci U S A 111:7456-7461. CrossRef Medline

Benes FM, Turtle M, Khan Y, Farol P (1994) Myelination of a key relay zone in the hippocampal formation occurs in the human brain during childhood, adolescence, and adulthood. Arch Gen Psychiatry 51:477-484. CrossRef Medline

Bullmore E, Sporns O (2009) Complex brain networks: graph theoretical analysis of structural and functional systems. Nat Rev Neurosci 10:186198. CrossRef Medline

Colizza V, Flammini A, Serrano MA, Vespignani A (2006) Detecting richclub ordering in complex networks. Nat Phys 2:110-115. CrossRef

Crossley NA, Mechelli A, Scott J, Carletti F, Fox PT, McGuire P, Bullmore ET (2014) The hubs of the human connectome are generally implicated in the anatomy of brain disorders. Brain 137:2382-2395. CrossRef Medline

Davey CG, Yücel M, Allen NB (2008) The emergence of depression in adolescence: development of the prefrontal cortex and the representation of reward. Neurosci Biobehav Rev 32:1-19. CrossRef Medline

Dennis EL, Jahanshad N, McMahon KL, de Zubicaray GI, Martin NG, Hickie IB, Toga AW, Wright MJ, Thompson PM (2013) Development of brain structural connectivity between ages 12 and 30: a 4-Tesla diffusion imaging study in 439 adolescents and adults. Neuroimage 64:671-684. CrossRef Medline

de Reus MA, van den Heuvel MP (2013) Rich club organization and intermodule communication in the cat connectome. J Neurosci 33:12929_ 12939. CrossRef Medline

Desikan RS, Ségonne F, Fischl B, Quinn BT, Dickerson BC, Blacker D, Buckner RL, Dale AM, Maguire RP, Hyman BT, Albert MS, Killiany RJ (2006) An automated labeling system for subdividing the human cerebral cortex on MRI scans into gyral based regions of interest. Neuroimage 31:968 980. CrossRef Medline

Di Martino A, Fair DA, Kelly C, Satterthwaite TD, Castellanos FX, Thomason ME, Craddock RC, Luna B, Leventhal BL, Zuo XN, Milham MP (2014) Unraveling the miswired connectome: a developmental perspective. Neuron 83:1335-1353. CrossRef Medline

Dosenbach NU, Nardos B, Cohen AL, Fair DA, Power JD, Church JA, Nelson SM, Wig GS, Vogel AC, Lessov-Schlaggar CN, Barnes KA, Dubis JW, Feczko E, Coalson RS, Pruett JR Jr, Barch DM, Petersen SE, Schlaggar BL 
(2010) Prediction of individual brain maturity using fMRI. Science 329: 1358-1361. CrossRef Medline

Dwyer DB, Harrison BJ, Yücel M, Whittle S, Zalesky A, Pantelis C, Allen NB, Fornito A (2014) Large-scale brain network dynamics supporting adolescent cognitive control. J Neurosci 34:14096-14107. CrossRef Medline

Fair DA, Dosenbach NU, Church JA, Cohen AL, Brahmbhatt S, Miezin FM, Barch DM, Raichle ME, Petersen SE, Schlaggar BL (2007) Development of distinct control networks through segregation and integration. Proc Natl Acad Sci U S A 104:13507-13512. CrossRef Medline

Fair DA, Cohen AL, Power JD, Dosenbach NU, Church JA, Miezin FM, Schlaggar BL, Petersen SE (2009) Functional brain networks develop from a "local to distributed" organization. PLoS Comput Biol 5:e1000381. CrossRef Medline

Fischl B (2012) FreeSurfer. Neuroimage 62:774-781. CrossRef Medline

Fischl B, Salat DH, Busa E, Albert M, Dieterich M, Haselgrove C, van der Kouwe A, Killiany R, Kennedy D, Klaveness S, Montillo A, Makris N, Rosen B, Dale AM (2002) Whole brain segmentation: automated labeling of neuroanatomical structures in the human brain. Neuron 33:341355. CrossRef Medline

Fornito A, Zalesky A, Bullmore ET (2010) Network scaling effects in graph analytic studies of human resting-state FMRI data. Front Syst Neurosci 4:22. CrossRef Medline

Fornito A, Zalesky A, Bassett DS, Meunier D, Ellison-Wright I, Yücel M, Wood SJ, Shaw K, O'Connor J, Nertney D, Mowry BJ, Pantelis C, Bullmore ET (2011) Genetic influences on cost-efficient organization of human cortical functional networks. J Neurosci 31:3261-3270. CrossRef Medline

Fornito A, Zalesky A, Breakspear M (2013) Graph analysis of the human connectome: promise, progress, and pitfalls. Neuroimage 80:426-444. CrossRef Medline

Fornito A, Zalesky A, Breakspear M (2015) The connectomics of brain disorders. Nat Rev Neurosci 16:159-172. CrossRef Medline

Giedd JN, Raznahan A, Alexander-Bloch A, Schmitt E, Gogtay N, Rapoport JL (2015) Child psychiatry branch of the national institute of mental health longitudinal structural magnetic resonance imaging study of human brain development. Neuropsychopharmacology 40: 43-49. CrossRef Medline

Grayson DS, Ray S, Carpenter S, Iyer S, Dias TG, Stevens C, Nigg JT, Fair DA (2014) Structural and functional rich club organization of the brain in children and adults. PLoS One 9:e88297. CrossRef Medline

Hagmann P, Sporns O, Madan N, Cammoun L, Pienaar R, Wedeen VJ, Meuli R, Thiran JP, Grant PE (2010) White matter maturation reshapes structural connectivity in the late developing human brain. Proc Natl Acad Sci U S A 107:19067-19072. CrossRef Medline

Harriger L, van den Heuvel MP, Sporns O (2012) Rich club organization of macaque cerebral cortex and its role in network communication. PLoS One 7:e46497. CrossRef Medline

Huttenlocher PR (1979) Synaptic density in human frontal cortex - developmental changes and effects of aging. Brain Res 163:195-205. CrossRef Medline

Hwang K, Velanova K, Luna B (2010) Strengthening of top-down frontal cognitive control networks underlying the development of inhibitory control: a functional magnetic resonance imaging effective connectivity study. J Neurosci 30:15535-15545. CrossRef Medline

Hwang K, Hallquist MN, Luna B (2013) The development of hub architecture in the human functional brain network. Cereb Cortex 23:2380-2393. CrossRef Medline

Jenkinson M, Bannister P, Brady M, Smith S (2002) Improved optimization for the robust and accurate linear registration and motion correction of brain images. Neuroimage 17:825-841. CrossRef Medline

Jenkinson M, Beckmann CF, Behrens TE, Woolrich MW, Smith SM (2012) FSL. Neuroimage 62:782-790. CrossRef Medline

Jeurissen B, Leemans A, Jones DK, Tournier JD, Sijbers J (2011) Probabilistic fiber tracking using the residual bootstrap with constrained spherical deconvolution. Hum Brain Mapp 32:461-479. CrossRef Medline

Jones DK, Knösche TR, Turner R (2013) White matter integrity, fiber count, and other fallacies: the do's and don'ts of diffusion MRI. Neuroimage 73:239-254. CrossRef Medline

Kelly AM, Di Martino A, Uddin LQ, Shehzad Z, Gee DG, Reiss PT, Margulies DS, Castellanos FX, Milham MP (2009) Development of anterior cingulate functional connectivity from late childhood to early adulthood. Cereb Cortex 19:640-657. CrossRef Medline
Lewis MD, Todd RM (2007) The self-regulating brain: cortical-subcortical feedback and the development of intelligent action. Cogn Dev 22:406430. CrossRef

Liston C, Watts R, Tottenham N, Davidson MC, Niogi S, Ulug AM, Casey BJ (2006) Frontostriatal microstructure modulates efficient recruitment of cognitive control. Cereb Cortex 16:553-560. CrossRef Medline

Lynall ME, Bassett DS, Kerwin R, McKenna PJ, Kitzbichler M, Muller U, Bullmore E (2010) Functional connectivity and brain networks in schizophrenia. J Neurosci 30:9477-9487. CrossRef Medline

Maslov S, Sneppen K (2002) Specificity and stability in topology of protein networks. Science 296:910-913. CrossRef Medline

Opsahl T, Colizza V, Panzarasa P, Ramasco JJ (2008) Prominence and control: the weighted rich-club effect. Phys Rev Lett 101:168702. CrossRef Medline

Panksepp J (1998) Affective neuroscience: the foundations of human and animal emotion. New York: Oxford UP.

Paus T (2010) Growth of white matter in the adolescent brain: myelin or axon? Brain Cogn 72:26-35. CrossRef Medline

Paus T, Collins DL, Evans AC, Leonard G, Pike B, Zijdenbos A (2001) Maturation of white matter in the human brain: a review of magnetic resonance studies. Brain Res Bull 54:255-266. CrossRef Medline

Paus T, Keshavan M, Giedd JN (2008) Why do many psychiatric disorders emerge during adolescence? Nat Rev Neurosci 9:947-957. CrossRef Medline

Ramirez-Manzanares A, Cook PA, Hall M, Ashtari M, Gee JC (2011) Resolving axon fiber crossings at clinical b-values: an evaluation study. Med Phys 38:5239-5253. CrossRef Medline

Reese TG, Heid O, Weisskoff RM, Wedeen VJ (2003) Reduction of eddycurrent-induced distortion in diffusion MRI using a twice-refocused spin echo. Magn Reson Med 49:177-182. CrossRef Medline

Rubinov M, Sporns O (2010) Complex network measures of brain connectivity: uses and interpretations. Neuroimage 52:1059-1069. CrossRef Medline

Schmithorst VJ, Yuan W (2010) White matter development during adolescence as shown by diffusion MRI. Brain Cogn 72:16-25. CrossRef Medline

Sowell ER, Thompson PM, Holmes CJ, Jernigan TL, Toga AW (1999) In vivo evidence for post-adolescent brain maturation in frontal and striatal regions. Nat Neurosci 2:859-861. CrossRef Medline

Spear LP (2000) The adolescent brain and age-related behavioral manifestations. Neurosci Biobehav Rev 24:417-463. CrossRef Medline

Steinberg L (2005) Cognitive and affective development in adolescence. Trends Cogn Sci 9:69-74. CrossRef Medline

Suckling J, Bullmore E (2004) Permutation tests for factorially designed neuroimaging experiments. Hum Brain Mapp 22:193-205. CrossRef Medline

Tau GZ, Peterson BS (2010) Normal development of brain circuits. Neuropsychopharmacology 35:147-168. CrossRef Medline

Tournier JD, Yeh CH, Calamante F, Cho KH, Connelly A, Lin CP (2008) Resolving crossing fibres using constrained spherical deconvolution: validation using diffusion-weighted imaging phantom data. Neuroimage 42:617-625. CrossRef Medline

Tournier JD, Calamante F, Connelly A (2012) MRtrix: diffusion tractography in crossing fiber regions. Int J Imaging Syst Technol 22:53-66. CrossRef

Towlson EK, Vértes PE, Ahnert SE, Schafer WR, Bullmore ET (2013) The rich club of the C. elegans neuronal connectome. J Neurosci 33:63806387. CrossRef Medline

Uddin LQ, Supekar KS, Ryali S, Menon V (2011) Dynamic reconfiguration of structural and functional connectivity across core neurocognitive brain networks with development. J Neurosci 31:18578-18589. CrossRef Medline

van den Heuvel MP, Sporns O (2011) Rich-club organization of the human connectome. J Neurosci 31:15775-15786. CrossRef Medline

van den Heuvel MP, Sporns O (2013) An anatomical substrate for integration among functional networks in human cortex. J Neurosci 33:1448914500. CrossRef Medline

van den Heuvel MP, Kahn RS, Goñi J, Sporns O (2012) High-cost, highcapacity backbone for global brain communication. Proc Natl Acad Sci U S A 109:11372-11377. CrossRef Medline

van den Heuvel MP, Sporns O, Collin G, Scheewe T, Mandl RC, Cahn W, Goñi J, Hulshoff Pol HE, Kahn RS (2013) Abnormal rich club organiza- 
tion and functional brain dynamics in schizophrenia. JAMA Psychiatry 70:783-792. CrossRef Medline

Wechsler D (1999) Wechsler Abbreviated Scale of Intelligence (WASI). San Antonio, TX: Harcourt.

Wechsler D (2003) Wechsler Intelligence Scale for children-4th Edition (WISC-IV). San Antonio, TX: Harcourt.

Whittle S, Yap MB, Yücel M, Fornito A, Simmons JG, Barrett A, Sheeber L, Allen NB (2008) Prefrontal and amygdala volumes are related to adolescents' affective behaviors during parent-adolescent interactions. Proc Natl Acad Sci U S A 105:3652-3657. CrossRef Medline

Xia M, Wang J, He Y (2013) BrainNet Viewer: a network visualization tool for human brain connectomics. PLoS One 8:e68910. CrossRef Medline
Yakovlev P, Lecours A (1967) The myelogenetic cycles of regional maturation of the brain. In: Regional development of the brain in early life (Minkowski A, ed), pp 3-70. Oxford: Blackwell Scientific.

Zalesky A, Fornito A, Bullmore ET (2010a) Network-based statistic: identifying differences in brain networks. Neuroimage 53:1197-1207. CrossRef Medline

Zalesky A, Fornito A, Harding IH, Cocchi L, Yücel M, Pantelis C, Bullmore ET (2010b) Whole-brain anatomical networks: does the choice of nodes matter? Neuroimage 50:970-983. CrossRef Medline

Zalesky A, Fornito A, Seal ML, Cocchi L, Westin CF, Bullmore ET, Egan GF, Pantelis C (2011) Disrupted axonal fiber connectivity in schizophrenia. Biol Psychiatry 69:80-89. CrossRef Medline 